\title{
Detection of Pressure and Heat in a Compressive Orthotic for Diabetes Prevention
}

\begin{abstract}
This paper presents a wearable system to detect the pressure and heat in a compressive orthotic and trigger an alarm when the values are beyond the standard margin of safety. In this way, the foot ulcers on diabetes' patients could be controlled and prevented. Arduino Nano is used as a microcontroller, two force sensors FSR402 and four temperature sensors LM35DZ are controlled by the microcontroller to detect the pressure and heat at different positions inside the compressive orthotic. Accuracy of the temperature sensor is $\pm 0.25^{\circ} \mathrm{C}$. A pair of Bluetooth $\mathrm{HC}-06$ is used to send data to computer. The threshold value of pressure and temperature difference is $3.38 \mathrm{~N}$ and $2.2{ }^{\circ} \mathrm{C}$ respectively. An alarm and warning LED will be triggered on the user interface based on LabVIEW. All the components are set on a printed circuit board and all the sensors are fixed on the inside of compressive orthotic as a wearable device.
\end{abstract}

Index Terms: Wearable, pressure detection, temperature sensors,

\section{INTRODUCTION}

Diabetes is a major health problem worldwide. Over the past decade, the number of patients who have diabetes increased from 1.08 hundred million in 1980 to 4.22 hundred million in 2014. The diabetes rate of adults who are older than 18 years old is raised from $4.7 \%$ to $8.5 \%$ [1]. One of the most serious problems linked to diabetes is that patients tend to develop foot ulcers. Ulcers appear because of the lack of sensation, poor blood circulation, feet deformities, and irritations due to diabetes. Diabetes foot has different symptoms, like foot ulcers, infection, and diseases of the blood vessel. Among these symptoms, foot ulcers are the most serial one, they can even lead to lower body part amputations which may really affect patients' lives. However, the symptoms of foot ulcers could be relieved or even avoided when detected and healed in the early stage of infection, the most popular methods to detect diabetes feet is temperature detection, pressure detection and peripheral vessels detection [3].

The compressive orthotic is used for treatment or prevention of different diseases by increasing the pressure of some special parts of the body to promote the blood circulation. Except treating diabetes foot, there are various compressive orthotics for different users and all have the positive effect on treatment. Some designed for treating diseases on other parts of body, like treating Asymmetric Pectus Carinatum, this compressive orthotic can be worn on patients' bodies [5] and apply force on a chest to control the angle of bones, then use radiographic marker to inspect the bone's structure when patients go the hospital for further examination or inspection [6]. Another compressive orthotic system is used on knees which can disperse the weight of the whole body to a larger part instead of a single point, thus heavy pressure could be adjusted to release the stress of knees which can speed up the process of recovery [7]. Besides, compressive orthotic could also be applied on hands, an electronic vibrating compression glove can supply compression and vibration therapy simultaneously to arthritic hands [8].

There are some similar compressive projects used on feet or limb, like stocking, which are used to prevent the hyperplasia afterburn, and Deep Vein Thrombosis [9] after surgery or cure foot ulcers led by diabetes. But the pressure applied by stocking is fixed, therefore, patients could only choose from 3 or 4 levels, and could not increase or decrease the pressure as they want. From the existent device introduced above, it is clearly understood that most compressive orthotics are focused on increasing the pressure and does not have a function to monitor the applied pressure and heat. However, patients do not know whether the pressure applied by compressive orthotic is too high or too low. The only way for patients to know the accurate conditions of their diseases inside the compressive orthotic is to do carry out the examination in the hospital.

For obtaining the actual conditions inside a compressive orthotic, a patent is designed for measuring the pressure points to be applied by a compressive orthotic device [10]. Since there are lots of patients suffered from diabetes, the smart application for healing and preventing foot ulcers by detecting the pressure become very popular among patients. High accuracy is the advantage of this patent. It measures many points of pressure on the whole leg and can finally display values in a pressure map. However, its utilization is limited by its low practicality, all the sensors are required when another patient needs to do the examination. Besides, patients and health professionals are required to work in the hospital together to detect the pressure due to the utilization of 
number of sensors and its considerable size, thus, making the whole operation inconvenient for both patients and professional.

The effectiveness of compressive orthotic will greatly depend on the pressure applied. The applied pressure can be divided into high and low levels, if the pressure is in a high level, it will deteriorate and the heat at the ulcers will be also be increased. In consequence, the setup can be hazardous and potentially dangerous, since no alarm will indicate a strain which is too high or too low. By detecting the pressure and heat in the compressive orthotic and sending a warning to other devices wirelessly, the problem could be solved, and the patients' complications and inflammations could be controlled in time. With the development of technology, cell phone, laptop or other portable devices are used everywhere. If wearable medical device could be designed to prevent foot ulcers and send data to patient's or professionals' portable devices, it will be much convenience for both patients and professionals in the hospital [11], through decreasing the rate of amputation and releasing the work load.

This paper introduces an innovative method to detect the pressure and heat in a compressive orthotic and trigger an alarm on a computer if the values that detected by the sensors are at a high level. Two force sensors and four temperature sensors are employed for obtaining data and connect the output to the microcontroller, and a pair of Bluetooth is used for serial communication with a computer. The values and warnings could be displayed directly on the user interface on the computer. This is a proof-of-concept study and it is aimed to extend using nano-sensors.

\section{SYSTEM DESIGN}

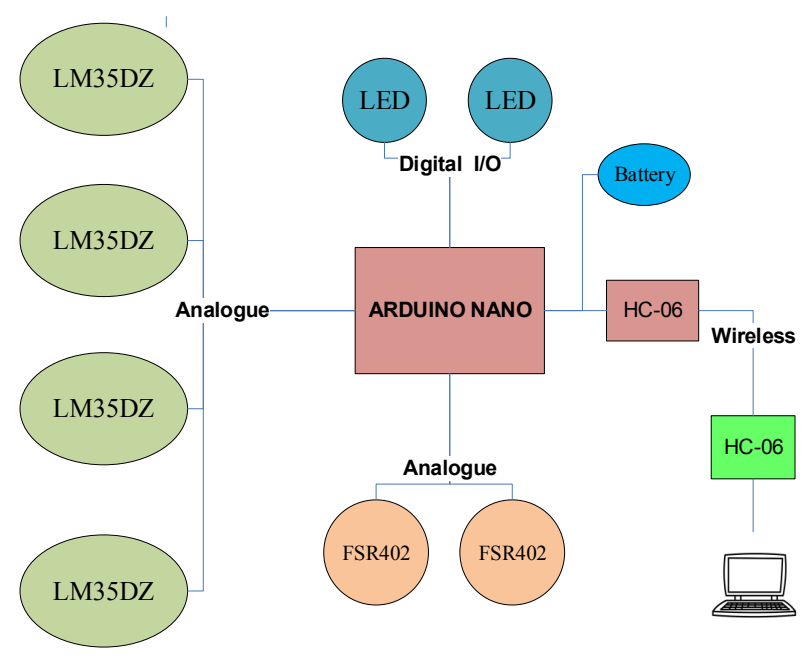

Fig1. Blocked diagram of the overall system

\section{A. PRESSURE DETECTION}

There are various kinds of products that which are employed by the health professionals in the hospital to increase the pressure on the limb, like compression stocking [9] and air pressure therapeutic equipment including therapies. Among these equipment's and therapies, the applied forces are different, but the force on the equipment that patient need to keep wearing is much smaller, according to analysis, $10 \mathrm{mmHg}-200 \mathrm{mmHg}$ is preferred. The force sensor's stressed area is a circle and it also has a tail which could be used to connect the other parts.

The stressed area $S$ of the force sensor FSR402 is:

$$
\mathrm{S}=\frac{\pi \mathrm{d}^{2}}{4}=\frac{3.14 \times 1.27^{2}}{4}=1.27 \mathrm{~cm}^{2}
$$

The intensity of minimum force $P_{-}$min and minimum force $F$ min that applied on fsr402 is given as:

$$
\begin{aligned}
& \text { P_min }=10 \mathrm{mmHg}=1.33 \mathrm{kpa}=0.01323 \mathrm{~N} / \mathrm{cm}^{2} \\
& \text { F_min }=0.01323 \mathrm{~N} / \mathrm{cm}^{2} \times 1.27 \mathrm{~cm}^{2}=0.168 \mathrm{~N}
\end{aligned}
$$

The intensity of maximum force $P_{-} \max$ and maximum force $F_{-}$max that applied on fsr402 is

$$
\begin{gathered}
\text { P_max }=200 \mathrm{mmHg}=26.66 \mathrm{kpa}=2.66 \mathrm{~N} / \mathrm{cm}^{2} \\
\text { F_max }=2.66 \mathrm{~N} / \mathrm{cm}^{2} \times 1.27 \mathrm{~cm}^{2}=3.38 \mathrm{~N}
\end{gathered}
$$

Range of the applied force beneficial to the patients from (3) and (5) is $0.168 \mathrm{~N}-3.38 \mathrm{~N}$. The range of force sensor fsr 402 is $0.1 \mathrm{~N}-100 \mathrm{~N}$ which is suitable for this use. When force is applied on the sensor, it displays values with a range from 0 to 1023 after ADC with supplied voltage $V c c=5 \mathrm{~V}$. Concerning the readability for patients and professional in the hospital, another $10 \mathrm{k} \Omega$ resistor is connected in series.

The relation between output voltage $V_{O U T}$ and resistance $R$ is given by:

$$
\mathrm{V}_{\mathrm{OUT}}=\frac{\mathrm{R}_{\mathrm{FSR} 402}}{\mathrm{R}_{\mathrm{FSR} 402}+10 \mathrm{k} \Omega} \times \mathrm{V}_{\mathrm{CC}}
$$

The force $F$ and resistance $R$ can be formulated as:

$$
\mathrm{F}=\left(6.25 / \mathrm{R}_{\mathrm{FSR} 402}\right)^{1.412}
$$

The relationship between force $\mathrm{F}$ and value after ADC is represented by:

$$
\mathrm{F}=\left(6.25 /\left(\frac{10 \mathrm{k} \times 1023-10 \mathrm{k} \times \text { value }}{\text { value }}\right)\right)^{1.412}
$$

\section{B. TEMPERATURE DETECTION}

The normal temperature of the human is $36-37^{\circ} \mathrm{C}$, but the skin temperature is different, trunk's temperature is always higher than limbs, because the blood circulation is faster near heart than other parts of the body. In addition, the external temperature will also affect the skin temperature, also the temperature in summer and winter has a significant difference [12]. If only use one threshold to warning the temperature will be subjective and may always have to change because of the factors mentioned above. It will be better to detect the temperature difference between the left side and right side of the limb at positions in the same height. According to the previous research, if the difference of the temperature at the same height is higher than 2.2 , it will have the risk to have an 
abnormal tissue, so the threshold of temperature is set to $2.2^{\circ} \mathrm{C}$.

LM35 is a temperature sensor which can work between $0{ }^{\circ} \mathrm{C}$ and $100{ }^{\circ} \mathrm{C}$ and do not need connect other external components with the accuracy in $\pm 1 / 4^{\circ} \mathrm{C}$. It has internal compensation, so it can work from 0 and can use without calibration and adjust [14]. The output voltage Vout is proportionate to the centigrade degree, $10 \mathrm{mV} /{ }^{\circ} \mathbf{C}$.

$$
\mathrm{V}_{\text {out }}=10 \mathrm{mV} /{ }^{\circ} \mathrm{C} \times \mathrm{T}^{\circ} \mathrm{C}
$$

The voltage supply from the microcontroller is $5 \mathrm{~V}$, so the formula for the temperature $T$ is

$$
\mathrm{T}=\frac{\text { value } \times \mathrm{V}_{\mathrm{CC}} \times 100}{1023}
$$

\section{BLUETOOTH COMMUNICATION}

Bluetooth is used to transmit the data from the microcontroller to computer. HC-06 is a Bluetooth that can be used when matching another Bluetooth. It has both 2 versions, with and without baseboard, the one that without baseboard can be embedded on the PCB with minimum space and the one with baseboard is connected to the computer through USB-TTL module. It can communicate with another Bluetooth within 10 meters and the Bluetooth on cellphone which is Android system can receive the data based on Bluetooth Specification V2.0. It can transmit data wirelessly only after setting up the Bluetooth through TTL serial port with AT instructions [15].

\section{PERFORMANCE AND RESULTS}

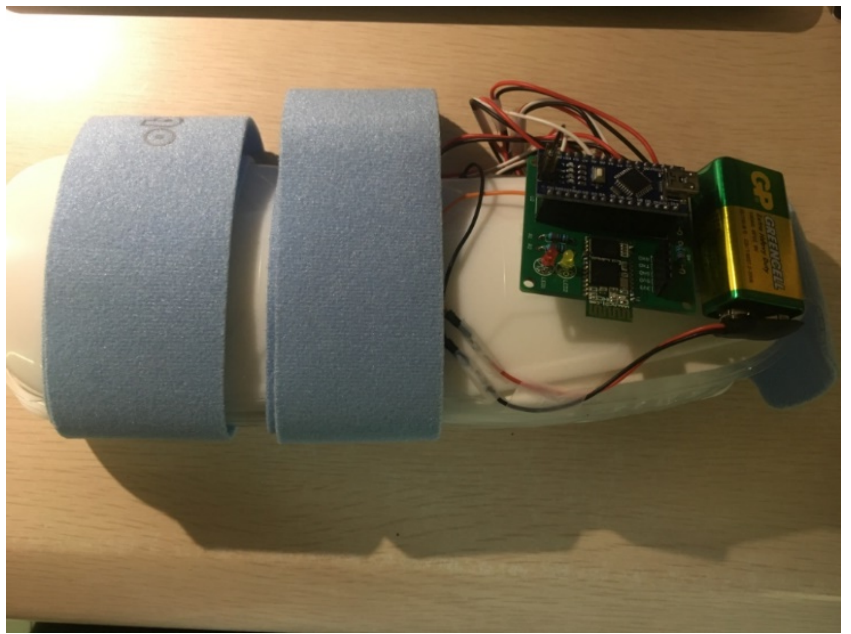

Figure 2. Experimental setup and implementation of the whole prototype

The actual project with Arduino Nano, two LEDs, Bluetooth, temperature and force sensors have to work together according to the program in Arduino and send values it detects to the user interface based on Lab VIEW to show the results wirelessly. In order to show the results and performance on the PCB and user interface based on the temperature and force sensors on the compressive orthotic, increasing the value of force as an example.

Figure 2 shows the performance on $\mathrm{PCB}$ and user interface in this condition.

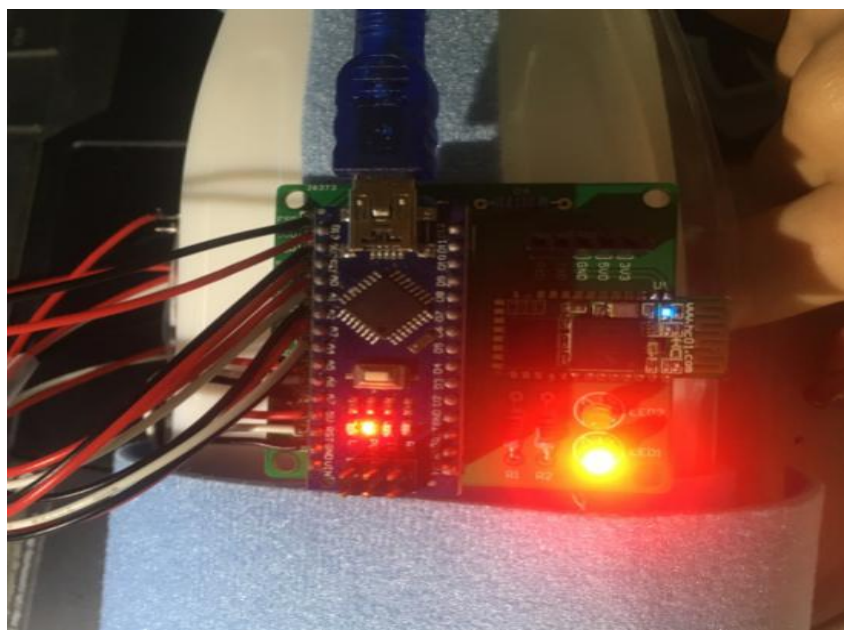

(a)

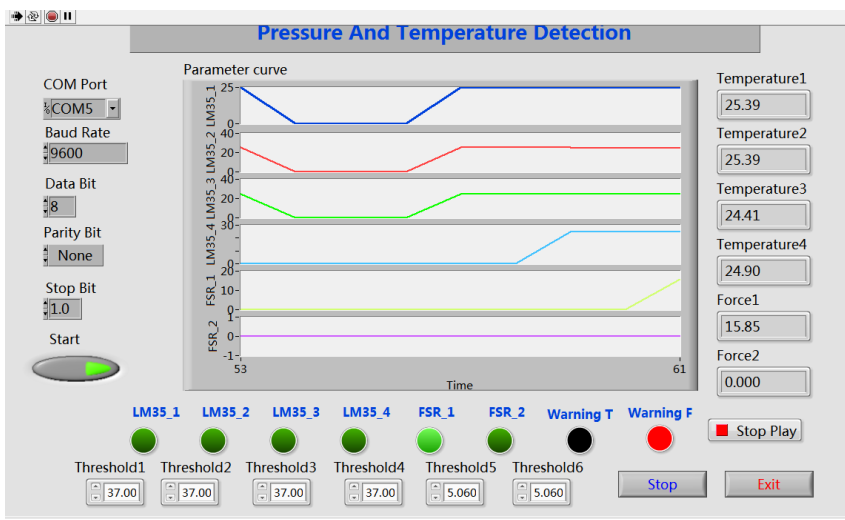

(b)

Figure 3. (a) The performance on PCB and (b) user interface when force is higher than $5.06 \mathrm{~N}$

When there is a power supply for Arduino, the LED on the Bluetooth will flash and will continue lighting if it is matched with another Bluetooth. The temperature and force values will display on the blocks at right side of the interface, and the waveforms will also be displayed. When increasing the pressure on force sensor 1 , the magnitude of the force is increased to 15.85 . It is higher than the threshold 5.06 set at the beginning of this software, at that time the corresponding analog LED will be illuminated. And because 15.85 is also beyond the threshold that set in the microcontroller, "Warning F" on the interface and LED1 on the PCB will also be illuminated. At the same time, an alarm will be triggered on the computer.

Likewise, when the temperature difference between sensors at the same height is higher than $2.2^{\circ} \mathbf{C}$, the "Warning T" LED on the interface and the LED2 on the PCB will be illuminated, and an alarm will be triggered. The difference between the pressure and temperature is the corresponding analogue LEDs on the user interface. 
The 4 analog LEDs on the interface will be illuminated when the each of the temperature is higher than its threshold which has nothing to do with the analogue "Warning T" and LED2 on the PCB.

\section{CONCLUSION}

In this paper, a novel method was introduced for the early detection of diabetes and its prevention. For the implementation of prototype, various components are combined such as force sensors, temperature sensors, LED, Bluetooth module, microcontroller together to detect pressure and heat in the compressive orthotic and trigger a LED and a warning on the user interface for diabetes prevention and its treatment. By using this approach, patients will be able to find out the infection parts well in time and draw their attention towards it to reduce the risk of having foot ulcers on diabetes' patients. It also gives an opportunity for patients to improve the process of recovery. It is predicted that, if it is widely used in daily treatment all around the world, both patients and professionals in the hospital could get benefit from it. These results have proved the viability of this system. Implementation of this system using nano-sensors is the future aspect of this work.

\section{ACKNOWLEDGMENT}

I would like to express my gratitude to my family, my friends for their help and also Glasgow College, UESTC, for providing the opportunity, components and laboratory.

\section{REFERENCES}

[1] Mathers, Colin D., and Dejan Loncar. "Projections of global mortality and burden of disease from 2002 to 2030." PLoS medicine 3, no. 11 (2006): e442.

[2] Li Ling, Zang Shasha, and Song Guangyao. " Risk factors and treatment progress of diabetes ulcers" Chinese General Practice 16.27(2013):3159-3163.

[3] Xu Zhangrong. "Classification and treatmnet progress of diabetes feet." Journal of Internal Medicine and critical Care 8.1(2002):32-35.

[4] Colagiuri S, Marsden L L, Naidu V, et al. "The use of orthotic devices to correct plantar callus in people with diabetes.” Diabetes Research \& Clinical Practice, 1995, 28(1):29-34.

[5] Berdan, Elizabeth A. "Compressive Orthotic Bracing: A Conservative Treatment Paradigm for Pectus Carinatum." In 2012 AAP National Conference and Exhibition. American Academy of Pediatrics, 2012.

[6] Egan J C, Dubois J J, Morphy M, et al. "Compressive orthotics in the treatment of asymmetric pectus carinatum: a preliminary report with an objective radiographic marker.". Journal of Pediatric Surgery, 2000, 35(8):1183-6.

[7] Matthews, Kevin C., and Alexander N. Leos. "Orthotic system and method utilizing hydrostatic compression of soft tissue to unload the knee and/or heel up to $100 \%$." U.S. Patent 9,427,348, August 30, 2016.

[8] Brown I E, Kounkel T L, Rodriguez B, et al. Electronic massaging orthotic compression glove[J]. 2017.
[9] Zheng Li,Yan Hu,Yifan Xue. "Incremental pressure stocking to prevent venous thromboembolism after operation [J]", Chinese Journal of Nursing, 2010, 45(5):478-480.

[10] Testud, J.L., Sennoune, M., Prudhomme, J.P. and Ouchene, A., Innothera Topic International SA, "Device for measuring pressure points to be applied by a compressive orthotic device". U.S. Patent 6,334,363. 1 Jan 2002

[11] Hung, K., Zhang, Y.T. and Tai, B., "Wearable medical devices for tele-home healthcare". In Engineering in Medicine and Biology Society, 2004. IEMBS'04. 26th Annual International Conference of the IEEE (Vol. 2, pp. 5384-5387).

[12] Wang Zheng, Bai Jiaojiao, Li Jing, "An investigation on feet skin temperature of diabetes group in community". CHINESE NURSING RESEARCH, 2008, 22(5):458-459.

[13] Melexis, MLX90614 family single and dual zone Infra red Thermometer in TO-39, www.melexis.com, June 29, 2015

[14] National Semiconductor, LM35 Precision Centigrade Temperature Sensors, accessed www.national.com, November 2000 [accessed on $13^{\text {th }}$ March 2018]

[15] Huicheng, HC-06 data sheet http://www.hc01.com/productdetail?productid=201701110 00 [accessed on $24^{\text {th }}$ March 2018] 